\title{
Cardiomiopatia Induzida por Estimulação Cardíaca Artificial
}

\author{
Cardiomyopathy Induced by Artificial Heart Stimulation
}

\author{
Silas dos Santos Galvão Filho ${ }^{1, *}$
}

Com o advento dos marcapassos cardíacos há mais de 60 anos, iniciou-se a era da estimulação cardíaca artificial, que mudou a história natural das bradicardias sintomáticas, aumentando significativamente a sobrevida principalmente dos pacientes portadores de bloqueio atrioventricular total. Inicialmente, os marcapassos eram monocâmaras ventriculares (VVI), trazendo algumas limitações em pacientes portadores de bradicardia consequente a disfunção sinusal, que na sua maioria apresentavam condução retrógrada ventrículo-atrial, determinando dissincronia atrioventricular com prejuízo na função cardíaca e consequente sintomas, o que foi chamado de síndrome do marcapassoํ․ No início dos anos 1980s, a incorporação de mais um eletro no átrio e o desenvolvimento dos marcapassos de dupla-câmara (DDD) também chamados na época de "marcapassos fisiológicos", resgataram o sincronismo atrioventricular e resolveram esse problema. Com o sucesso dos marcapassos de dupla câmara, passou-se a imaginar que eles poderiam aumentar ainda mais a sobrevida dos seus portadores quando comparados aos VVI. Entretanto, apesar dos marcapassos DDD mostrarem benefícios na tolerância aos esforços e melhora na qualidade de vida, não foi evidenciado significativo aumento da sobrevida nessa comparação.

Nos primeiros anos do segundo milênio, concomitante ao aparecimento da terapia de ressincronização cardíaca (TRC) e os conceitos de dissincronia, passou-se a observar que pacientes portadores de dispositivos de dupla câmara que apresentavam estimulação ventricular efetiva em mais de $40 \%$ dos batimentos cardíacos apresentavam alguns problemas, tais como: aparecimento ou piora de insuficiência cardíaca, desenvolvimento de fibrilação atrial e até mesmo aumento de mortalidade ${ }^{2-4}$ quando comparados aos que não apresentavam estimulação de $\mathrm{VD}$, sendo isso tanto pior quanto maior a percentagem de estimulação ventricular. Esses problemas foram atribuídos à dissincronia ventricular causada pela estimulação de VD e levaram ao desenvolvimento, nos dispositivos de estimulação cardíaca artificial, de algoritmos programáveis de mínima estimulação ventricular, com objetivo de evitar estimulações ventriculares desnecessárias em pacientes que apresentassem condução atrioventricular e interventricular preservadas.

Já nos casos de bloqueios atrioventriculares, em que a estimulação de VD é absolutamente necessária, passou-se a procurar novos sítios de estimulação opcionais à clássica estimulação de ponta de VD. Nesse sentido, vários sítios de estimulação ventricular foram tentados: Via de saída do VD, região médio septal, ínfero-septal, sem, entretanto, mostrarem diferenças significativas quando comparados à estimulação clássica da ponta do $\mathrm{VD}^{5,6}$.

Nos últimos anos, o desenvolvimento de bainhas e eletrodos especiais para estimulação do tronco do feixe de His possibilitaram essa modalidade de estimulação cardíaca que preserva a condução interventricular através do sistema de condução normal, evitando consequentemente a dissincronia ventricular induzida pela estimulação de $\mathrm{VD}^{7,8}$. Essa modalidade de estimulação entretanto, tem sua indicação nos casos que apresentem condução intraventricular preservada através do sistema de condução normal, pois nos casos em que existam distúrbios de condução intraventricular como o bloqueio do ramo esquerdo (BRE), a estimulação do tronco do feixe de His não traz benefícios, já que determina a persistência do distúrbio de condução e, consequentemente, a dissincronia. Mais recentemente, utilizando-se o mesmo sistema desenvolvido para estimulação de His, passou-se a estimular a região muscular

1.Centro Avançado de Ritmologia e Eletrofisiologia - São Paulo (SP), Brasil.

Recebido: Mar. 10, 2020 | Aceito: Mar. 20, 2020

*Autor correspondente: sdsantos@uol.com.br

ORCID: Galvão Filho SS (iD https://orcid.org/0000-0002-5152-2648 
do septo interventricular, imediatamente abaixo da porção fibrótica onde se encontra o tronco do feixe de His ${ }^{9}$, penetrando no septo interventricular e estimulando as porções iniciais dos ramos do feixe de His, corrigindo em muitos casos os distúrbios de condução como o BRE. Essa promissora modalidade de estimulação cardíaca artificial tem se colocado como opção de estimulação unissítio à TRC com estimulação biventricular, apesar de ainda necessitar de maior conhecimento dos seus resultados a longo prazo.

A nossa ideia de que a dissincronia ventricular poderia induzir miocardiopatia dilatada partiu de um trabalho que apresentamos em 1997 no Congresso Brasileiro de Arritmias Cardíacas ${ }^{10}$, onde foi apresentado um estudo com 12 pacientes portadores de cardiomiopatia dilatada idiopática que apresentavam dissincronia ventricular (BRE), fração de ejeção do ventrículo esquerdo (FEVE) reduzida (<35\%) e ICC refratária a terapêutica medicamentosa, que ,após TRC, normalizaram a função sistólica cardíaca com normalização da FEVE. Em três desses pacientes houve normalização de todos os parâmetros ecocardiográficos com o exame mostrando coração estruturalmente normal. Atribuímos à dissincronia determinada pelo BRE a causa do aparecimento da cardiomiopatia dilatada tida como idiopática, que chamamos de "dessincroniomiopatia".

A estimulação convencional de VD determina padrão eletrocardiográfico de BRE, com largura do QRS frequentemente superior a 150 ms e alterações da contratilidade miocárdica ventricular (dissincronia) similares ao BRE, com prejuízo da função sistólica cardíaca ${ }^{11}$. Em alguns (mas não em todos) pacientes, essas alterações podem ser responsáveis pelo aparecimento e/ ou agravamento de insuficiência cardíaca e até mesmo alterações estruturais do coração, podendo, portanto, ser responsáveis pelo desenvolvimento de "dessincroniomiopatia". Esses pacientes se beneficiam muito do chamado upgrade para TRC, com implante de mais um eletrodo para estimulação VE.

Gage et al. ${ }^{12}$ publicaram trabalho em que compararam a resposta clínica e ecocardiográfica à TRC de pacientes com ou sem estimulação cardíaca convencional prévia (ECCP), sendo demonstrado uma resposta clínica positiva similar entre os dois grupos e uma melhor resposta dos pacientes com ECCP com relação à função ventricular avaliada pelo eco. Além disso foi demonstrada uma tendência de menor mortalidade dos pacientes com ECCP quando comparados aos sem. Os autores concluíram que pacientes submetidos a upgrade de marcapasso para TRC apresentam uma resposta similar, se não melhor, que a TRC em pacientes sem ECCP.

Vários estudos como o de Martineli et al. ${ }^{13}$ mostraram melhor resultado do implante de TRC quando comparados ao marcapasso convencional em pacientes com indicação de estimulação ventricular e função sistólica ventricular reduzida. Recentemente, foi publicada uma revisão sistemática ${ }^{14}$ envolvendo 679 pacientes de oito estudos que avaliaram o impacto do que chamaram de estimulação cardíaca fisiológica (ECF) (TRC ou estimulação de His/ParaHis) versus estimulação cardíaca convencional em pacientes com indicação de marcapassos cardíacos e função sistólica ventricular moderadamente comprometida (> 35\%), tendo sido demonstrado significativa superioridade da ECF em relação aos parâmetros ecocardiográficos (FEVE, volume diastólico final do VE, volume sistólico final do VE).

Nas diretrizes da Sociedade Europeia de Cardiologia (ESC) para manejo da ICC 2016 ${ }^{15}$, a TRC tem indicação classe 1 nos pacientes com indicação de estimulação cardíaca que necessitem de estimulação de $\mathrm{VD}$ e apresentem $\mathrm{FE}<40 \%$, em detrimento dos marcapassos convencionais. Nessas diretrizes, o upgrade de marcapasso para TRC tem indicação IIA nos casos de portadores de disfunção sistólica $(\mathrm{FEVE}<40 \%)$ que apresentarem piora da ICC após implante de dispositivo com estimulação de VD em mais de 40\% dos batimentos cardíacos.

Apesar de ter a possiblidade de causar prejuízos na função sistólica cardíaca em alguns pacientes, podendo até mesmo ser responsável pelo aparecimento/agravamento de cardiomiopatias dilatadas chamadas por nós de "dessincroniomiopatias", a estimulação convencional unissítio de VD persiste sendo utilizada rotineiramente. A boa evolução da grande maioria dos portadores de marcapassos convencionais, a maior complexidade dos procedimentos para estimulação biventricular e até mesmo os custos bem maiores dos dispositivos de TRC limitam a utilização mais frequente dessa modalidade de estimulação cardíaca em pacientes com indicação de marcapassos que necessitam de estimulação ventricular. A estimulação transeptal das porções iniciais dos ramos do feixe de His pode vir a ser uma excelente opção de estimulação unissítio de VD à TRC com estimulação biventricular.

Tendo-se o conhecimento dos possíveis prejuízos causados pela estimulação unissítio de VD dos marcapassos convencionais, os portadores desses dispositivos devem ser avaliados em relação à parte estrutural e funcional do coração, através de métodos de imagem como o ecocardiograma, no mínimo anualmente e, caso mostrem alterações relevantes e/ ou progressivas, o upgrade para TRC deve sempre ser considerado. 


\section{REFERÊNCIAS}

1. Mitsue T, Hori M, Suma K et al. The "pacemaker syndrome". In: Jacob JE (ed). Proceedings of the Right Annual International Conference on Medical and Biological Engineering. Chicago: Association for the Advancement of Medical Instrumentation; 1969. 29-33 p.

2. The DAVID Trial Investigators. Dual-chamber pacing or ventricular backup pacing in patients with and implantable defibrillator: The dual-chamber and WI implantable defibrillator (DAVID) trial. JAMA. 2002;288(24):3115-23. https://doi.org/10.1001/jama.288.24.3115

3. Sweeney MO, Helkamp AS, Ellenboge KA, Greenspon AJ, Freedman RA, Lee KL et al. Mode selection trial. Adverse effect of ventricular pacing on heart failure and atrial fibrillation among patients with normal baseline QRS duration in a clinical trial of pacemaker therapy for sinus node dysfunction. Circulation. 2003;107(23):2932-7. https://doi.org/10.1161/01.CIR.0000072769.17295.B1

4. Nielsen JC, Kristensen L, Andersen HR, Mortensen PT, Pedersen OL, Pedersen AK. A randomized comparation of atrial and dualchamber pacing in 17 consecutive patients with sick sinus syndrome: echocardiographic and clinic outcome. J Am Coll Cardiol. 2003;42(4):614-23. https://doi.org/10.1016/S0735-1097(03)00757-5

5. Zografos TA, Siontis KC, Jastrzebski M, Kutyifa V, Klein HU, Zareba W, et al. Apical vs. non-apical right ventricular pacing in cardiac resynchronization therapy: a meta-analysis. EP Eur. 2015;17(8):1259-66. https://doi.org/10.1093/europace/euv048

6. Shimony A, Eisemberg MJ, Filion KB, Amit G. Beneficial effects of right ventricular non-apical vs. apical pacing: a systematic review and meta-analysis of randomized-controlled trials. EP Eur. 2012;14(1):81-91. https://doi.org/10.1093/europace/eur240

7. Vijayaraman P, Chung MK, Dandamudi G, Upadhyay GA, Krishnan K, Grossley G, et al. ACC's Electrophysiology Council. His Bundle Pacing. J Am Coll Cardiol. 2018;72(8):927-47.

8. Ali N, Kreene D, Arnold A, Shun-Shin M, Whinnett ZI, Sohaib A. His bundle pacing: a new frontier in the treatment of heart failure. Arrhythm Electrophysiol Rev. 2018;7(2):103-10. https://doi.org/10.15420/aer.2018.6.2

9. Upadhyay GA, Cherian T, Shatz DY, Beazer AD, Aziz Z, Ozcan C, et al. Intracardiac delineation of septal conduction in left bundlebranch block patterns. Circulation. 2019;139(16):1876-88. https://doi.org/10.1161/CIRCULATIONAHA.118.038648

10. Sacramento ERS, Galvão Filho SS, Vasconcelos JTM, Leobino E, Fragata C, Barcelos C. Normalização da função sistólica ventricular na cardiomiopatia dilatada idiopática pós ressincronização cardíaca. Tratamento da "dissincroniomiopatia". Relampa. 2007;20(4):272.

11. Bank AJ, Schwarzman DS, Burns KV, Kaufman CL, Adler SW, Kelly AS, et al. Intramural dyssynchrony from acute right ventricular apical pacing in human subjects with normal left ventricular function. J Cardiovasc Trans Res. 2010;3:321-29. https://doi.org/10.1007/s12265-010-9176-8

12. Gage RM, Burns KV, Bank AJ. Echocardiographic and clinical response to cardiac resynchronization therapy in heart failure patients with and without previous right ventricular pacing. Eur J Heart Fail. 2014;16(11):1199-205. https://doi.org/10.1002/ejhf.143

13. Martinelli Filho M, Siqueira SF, Costa R, Greco OT, Moreira LF, D'Avila A, et al. Conventional versus biventricular pacing in heart failure and bradyarrhythmia: the COMBAT study. J Cardiac Fail. 2010;16(4):293-300. https://doi.org/10.1016/j.cardfail.2009.12.008

14. Slotwiner DJ, Rait MH, Munoz FD-C, Mulpuru SK, Nasser N, Peterson PN. Impact of physiologic pacing versus right ventricular pacing among patients with left ventricular ejection fraction greater than 35\%: a systematic review for the 2018 ACC/AHA/HRS guidelines on the evaluation and management of patients with bradycardia and cardiac conduction delay. A report of the American College of Cardiology/American Heart Association Task Force on Clinical Practice Guidelines and the Heart Rhythm Society. Circulation. 2019;140(8):e483-503. https://doi.org/10.1161/CIR.0000000000000722

15. Ponikowski P, Voors AA, Anker SD, Bueno H, Cleland JG, Coats AJ, et al. 2016 ESC Guidelines for the diagnosis and treatment of acute and chronic heart failure: The Task Force for the diagnosis and treatment of acute and chronic heart failure of the European Society of Cardiology (ESC). Developed with the especial contribution of the Heart Failure Association (HFA) of the ESC. Eur J Heart Fail. 2016;18(8):891-975. 